\title{
Loss of mTOR repressors Tsc1 or Pten has divergent effects on excitatory and inhibitory synaptic transmission in single hippocampal neuron cultures
}

\author{
Matthew C. Weston ${ }^{1,2}$, Hongmei Chen ${ }^{1,2}$ and John W. Swann ${ }^{1,2} *$ \\ ${ }^{1}$ The Cain Foundation Laboratories, The Jan and Dan Duncan Neurological Research Institute, Houston, TX, USA \\ 2 Departments of Neuroscience and Pediatrics, Baylor College of Medicine, Houston, TX, USA
}

\section{Edited by:}

Bryan Weston Luikart, Geisel School

of Medicine at Dartmouth, USA

\section{Reviewed by:}

Helen S. Bateup, Harvard Medical School, USA

Jacek Jaworski, International Institute of Molecular and Cell Biology, Poland

\section{*Correspondence:}

John W. Swann, The Cain Foundation Laboratories, The Jan and Dan Duncan Neurological Research Institute, 1250 Moursund Street, Suite 1225, Houston, TX 77030, USA e-mail: jswann@bcm.edu
The Pten and Tsc1 genes both encode proteins that repress mechanistic target of rapamycin (mTOR) signaling. Disruption of either gene in the brain results in epilepsy and autism-like symptoms in humans and mouse models, therefore it is important to understand the molecular and physiological events that lead from gene disruption to disease phenotypes. Given the similar roles these two molecules play in the regulation of cellular growth and the overlap in the phenotypes that result from their loss, we predicted that the deletion of either the Pten or TsC1 gene from autaptic hippocampal neurons would have similar effects on neuronal morphology and synaptic transmission. Accordingly, we found that loss of either Pten or Tsc1 caused comparable increases in soma size, dendrite length and action potential properties. However, the effects of Pten and Tsc1 loss on synaptic transmission were different. Loss of Pten lead to an increase in both excitatory and inhibitory neurotransmission, while loss of Tsc 1 did not affect excitatory neurotransmission and reduced inhibitory transmission by decreasing mIPSC amplitude. Although the loss of Pten or Tsc1 both increased downstream mTORC1 signaling, phosphorylation of Akt was increased in Pten-ko and decreased in Tsc1-ko neurons, potentially accounting for the different effects on synaptic transmission. Despite the different effects at the synaptic level, our data suggest that loss of Pten or Tsc1 may both lead to an increase in the ratio of excitation to inhibition at the network level, an effect that has been proposed to underlie both epilepsy and autism.

Keywords: pten, TSC/mTOR, synaptic transmission, akt, excitatory transmission, inhibitory transmission, epilepsy, autism

\section{INTRODUCTION}

The evolutionarily conserved mTOR signaling network integrates information from growth factor receptor activity, ATP, glucose, and amino acid levels to control cellular growth, proliferation, and autophagy (Sarbassov et al., 2005a; Wullschleger et al., 2006; Laplante and Sabatini, 2012). In line with these essential cellular functions, mTOR dysfunction is associated with a broad range of human diseases including cancer, type II diabetes and obesity, and major efforts to develop drugs targeting the pathway are underway (Guertin and Sabatini, 2009). In the brain, hyperactivation of mTOR signaling causes neurological disorders in both humans and in animal models. Although dependent on the mutation, the region affected and the extent of expression, the most common symptoms are epilepsy, autism, and intellectual disability (Swiech et al., 2008; Ehninger et al., 2009; Hoeffer and Klann, 2009; Russo et al., 2012).

Two established genetic mechanisms through which mTOR signaling abnormalities lead to neurological disease are mutations that disrupt the Pten or Tscl genes. The protein products of these genes are similar in many ways: (1) they are both repressors of mTOR signaling and in their absence the mTORC1 complex is hyperactive, (2) their loss in neurons can lead to hyperexcitable network activity, and (3) rapamycin, an inhibitor of mTOR, blocks the cellular and behavioral consequences of their loss in animal models, and potentially humans as well (Krueger et al., 2013). This evidence predicts that the effects of Pten or Tsc1/2 disruption on neuronal form and function may be similar.

At the molecular level, studies from yeast and in mammalian cell lines, mostly in the context of cancer research, have provided a wealth of information on the basic organization of the mTOR pathway and the roles that Pten and Tsc1 play. From these studies it is clear that one of the major inputs to mTOR activity is insulin and growth factor receptor signaling (Harris and Lawrence, 2003; Manning and Cantley, 2003; Avruch et al., 2006; Howell and Manning, 2011). Receptor activation, through a series of intracellular events utilizing phosphoinositide-3 kinase (PI3K), leads to increased PIP 3 levels and membrane localization of Akt, a state that favors its phosphorylation. Pten represses this pathway by catalyzing the conversion of $\mathrm{PIP}_{3}$ to $\mathrm{PIP}_{2}$, which ultimately leads to reduced Akt phosphorylation and reduced activity of Akt toward its substrates. One important substrate of Akt is Tsc2, which complexes with Tscl to function as a GTPase activating protein that, via Rheb, inhibits mTORC1. Loss of either Pten or Tsc1 are both thought to disrupt these molecular events and lead to excessive activity of mTORC1, however, the unique position of each 
protein in the pathway and the potential of each to act on targets other than their canonical mTOR-related ones raise the possibility that the functional consequences of their loss may not be overlapping.

In neurons, previous studies have consistently shown that loss of either Pten or Tsc1/2 increases neuronal cell body size (Backman et al., 2001; Kwon et al., 2001; Tavazoie et al., 2005; Meikle et al., 2007; Feliciano et al., 2011). Loss or reduction of Pten also increases growth of axons, dendrites, and spine/synapse formation (Jaworski et al., 2005; Kwon et al., 2006; Fraser et al., 2008). While loss of Tsc1/2 has been shown to increase axon growth and dendritic complexity (Meikle et al., 2007; Choi et al., 2008; Feliciano et al., 2011,2012), the reported effects on spine and synapse number are variable (Tavazoie et al., 2005; Meikle et al., 2007; Bateup et al., 2011; Tsai et al., 2012). From a physiological standpoint, loss of Pten has been shown to increase excitatory synaptic transmission due to increases in the mEPSC amplitude (Luikart et al., 2011; Weston et al., 2012; Xiong et al., 2012). While loss of Tsc1 has also been linked to increased excitation, the underlying mechanism is not clear (Tavazoie et al., 2005; Wang et al., 2007; Bateup et al., 2011, 2013). Differences in neuron type, time, and extent of gene deletion and secondary effects of seizures may all have contributed to these variable results.

In order to test the extent to which loss of Pten or Tsc1 has overlapping effects on neuronal form and function, we performed a direct comparison of the cellular neurophysiology and morphology of autaptic hippocampal neurons in which Pten or Tsc1 was genetically disrupted. We found that loss of Pten or Tsc1 caused similar changes in neuronal morphology, passive membrane, and action potential properties and downstream mTORC1 signaling. The effects of loss of Pten or Tsc1 on synaptic transmission were, however, different. Loss of Pten increased excitatory neurotransmission, while loss of Tsc1 had no effect. Loss of Pten also increased inhibitory synaptic transmission, while loss of Tscl decreased it, suggesting that, despite their many similarities, loss of Pten and Tsc1 may lead to a disruption of the excitatory-inhibitory balance in the hippocampus through distinct mechanisms.

\section{RESULTS}

In order to directly compare the cellular effects of Pten or Tsc1 loss, we made primary cultures of hippocampal neurons from either $\mathrm{Pten}^{\text {loxP/loxP }}$ or $\mathrm{Tsc} 1^{\text {loxP } / \mathrm{loxP}}$ mice and infected them with a lentivirus expressing a Cre recombinase-RFP fusion protein (hereafter referred to as Pten-ko and Tsc1-ko neurons). Control neurons were either loxP/loxP neurons from the same animal infected with a control virus or wild-type littermates infected with the Cre virus. The cultures were single-neuron (autaptic) cultures on astrocyte islands. This preparation allowed us to simultaneously test both pre- and postsynaptic function, to quantify several electrophysiological and morphological parameters on a per cell basis, and to examine the effects of Pten and Tsc1 loss in the absence of network or activity-dependent effects.

We verified that the cre-mediated gene deletion led to expected increases in mTOR signaling by testing for increased levels of phospho-S6 (pS6) and phospho-4E binding protein (p4E-BP), as well as increased soma size, which are three well-characterized consequences of hyperactive mTOR signaling (Figure 1). Using quantitative immunofluorescence analysis of both Pten- and Tsc1deficient single neurons (Figure 1A), we found that the loss of either Pten or Tscl caused significant increases in both pS6 and $\mathrm{p} 4 \mathrm{E}-\mathrm{BP}$ immunofluorescence intensity relative to controls (Figure 1C). We also immunostained for Map2, to visualize the soma of Pten- and Tscl-ko neurons. We then imaged the neurons, traced the cell bodies using Neurolucida software and measured their area. The area of the cell body was significantly increased in both Pten- and Tsc-ko neurons (Figure 1C). Finally, to verify that the changes in pS6 levels and soma size were due to hyperactive mTOR in both mutants, we treated the neurons with $20 \mathrm{nM}$ rapamycin at DIV 7 for $72 \mathrm{~h}$ and repeated the pS6, p4EBP, and cell size measurements. Rapamycin treatment reduced both pS6 levels and soma area in both Pten and Tsc1-ko neurons to control levels or lower (Figures 1B,C). p4E-BP levels in Pten and Tsc1-ko neurons were not decreased by rapamycin treatment (Figure 1C), in agreement with previously reported effects of longterm rapamycin treatment on $4 \mathrm{E}-\mathrm{BP}$ phosphorylation in some cell types (Choo et al., 2008; Thoreen et al., 2009).

\section{LOSS OF Pten OR Tsc1 AFFECTS PASSIVE MEMBRANE AND ACTION POTENTIAL PROPERTIES}

Previous electrophysiological studies of both Pten and Tsc-lacking neurons have consistently shown effects on passive membrane properties, especially membrane capacitance, consistent with the large influence that the mTOR signaling pathway has on regulation of cellular growth (Bateup et al., 2011, 2013; Luikart et al., 2011; Weston et al., 2012; Xiong et al., 2012; Normand et al., 2013). In order to verify this effect in our system, and to compare the magnitude of the changes induced by a loss of Pten or Tsc1, we performed a whole cell patch current clamp analysis of the neurons (Figure 2; Table 1). The resting membrane potentials $\left(V_{\mathrm{m}}\right)$ of Pten- and Tsc1-ko neurons were not different from their respective controls (Figure 2B). Injection of hyperpolarizing current steps and then measuring the time course and extent of the resulting voltage changes revealed that neither Pten- nor Tsc1-ko neurons had altered membrane time constants, but that the input resistance was significantly decreased to similar levels for both knockouts (Figure 2B). As a result, the calculated membrane capacitance was also increased to a similar extent for both Pten- and Tsc1-ko neurons vs. control (Figure 2B).

Next, we injected depolarizing current steps to examine the action potential (AP) properties of Pten- and Tsc1-ko neurons. The AP threshold was not different in either Pten- or Tsc1-ko neurons, however, there were significant effects on the shape of the AP in both Pten- and Tsc1-ko neurons (Figure 2C). Both mutants had action potentials that were smaller in amplitude and with a greater duration at half the maximal amplitude (AP halfwidth) than their controls (Figures 2C,D). Despite the reduced input resistance, there was no difference in the amount of injected current needed to elicit an AP (Figure 2D), or the number of APs for a given current injection (Figure 2A).

\section{LOSS OF Pten INCREASES AP EVOKED GLUTAMATERGIC TRANSMISSION BUT LOSS OF TSC1 DOES NOT}

Previous studies of both Pten- and Tscl-ko mice have suggested that altered excitatory transmission in these models is an 


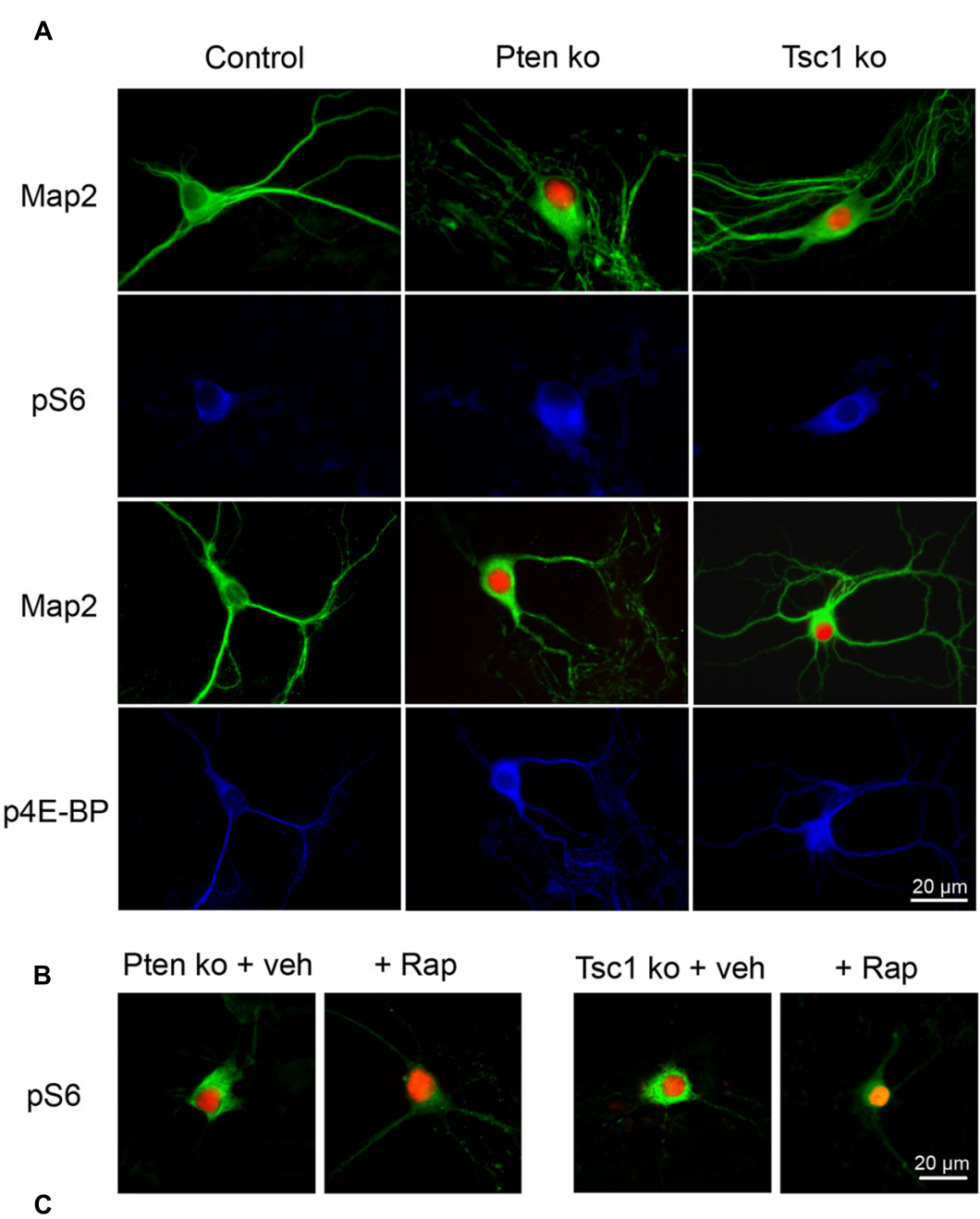

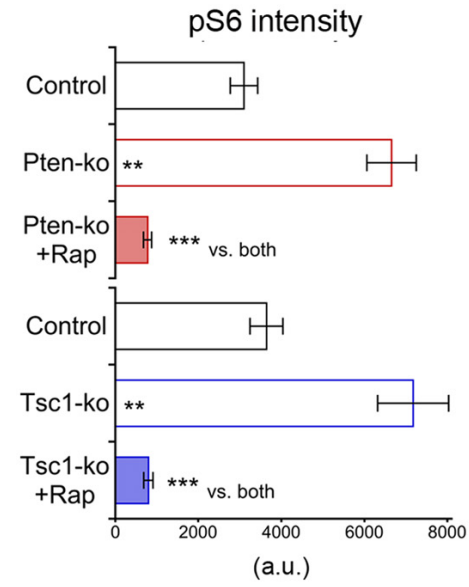

FIGURE 1 | Cre-mediated deletion of Pten or Tsc1 causes mTORC1 activation and an increase in cell size. (A) Representative images showing immunofluorescence from Map2 (green) and either phospho-S6 or phospho-4E-BP (blue) from control (left column), Pten-ko (middle column) and Tsc1-ko (right column) neurons. Red fluorescence is shown in Pten- and Tsc1-ko neurons from the Cre-RFP fusion protein. (B) Representative images showing immunofluorescence from phospho-S6

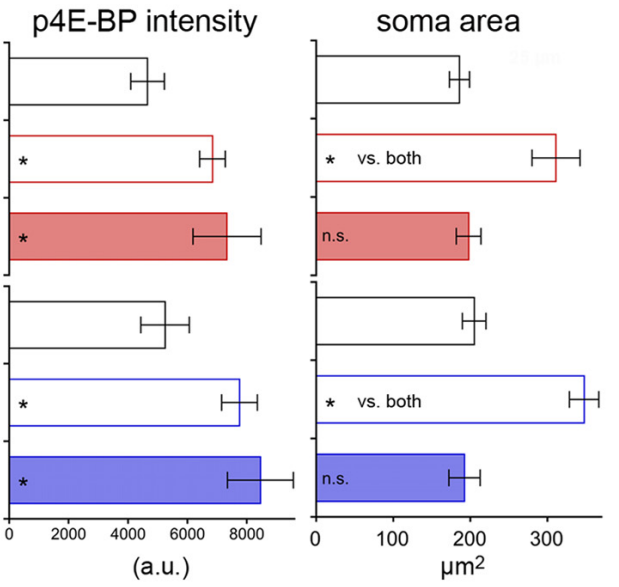

(green) and the Cre-RFP fusion protein from Pten-ko (left images) and Tsc1-ko (right images) treated with either DMSO vehicle or $20 \mathrm{nM}$ rapamycin for 72 h. (C) Summary data showing the changes in pS6, p4E-BP and soma area (mean \pm SEM) for control, knockout and rapamycin-treated knockout neurons for Pten (red bars) and Tsc1 (blue bars). ${ }^{*} p \leq 0.05, * * p \leq 0.01,{ }^{* * *} p \leq 0.001$, n.s. $=$ not significant vs. control unless indicated. 


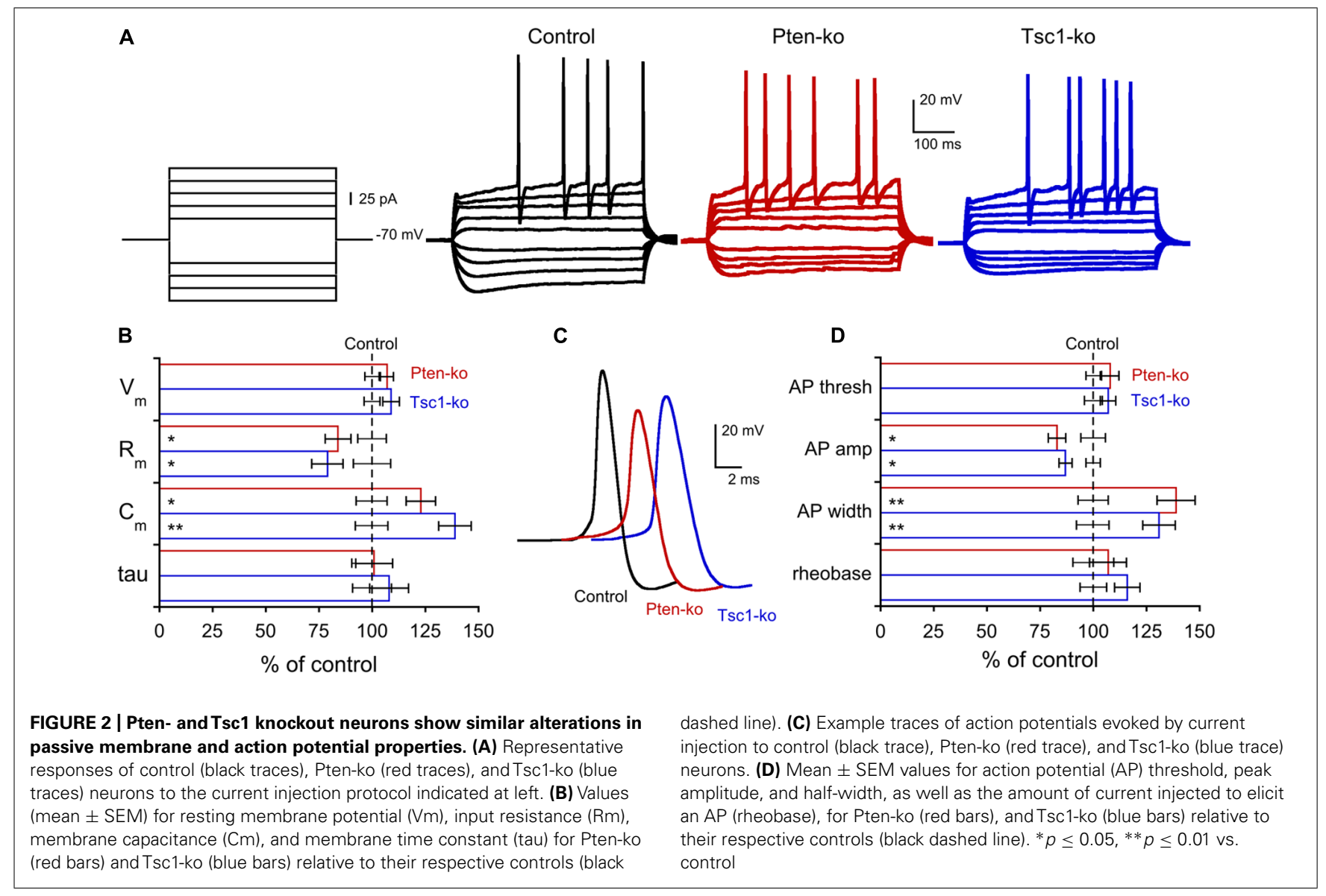

Table 1 | Passive membrane and AP properties of Pten- and Tsc1-ko neurons.

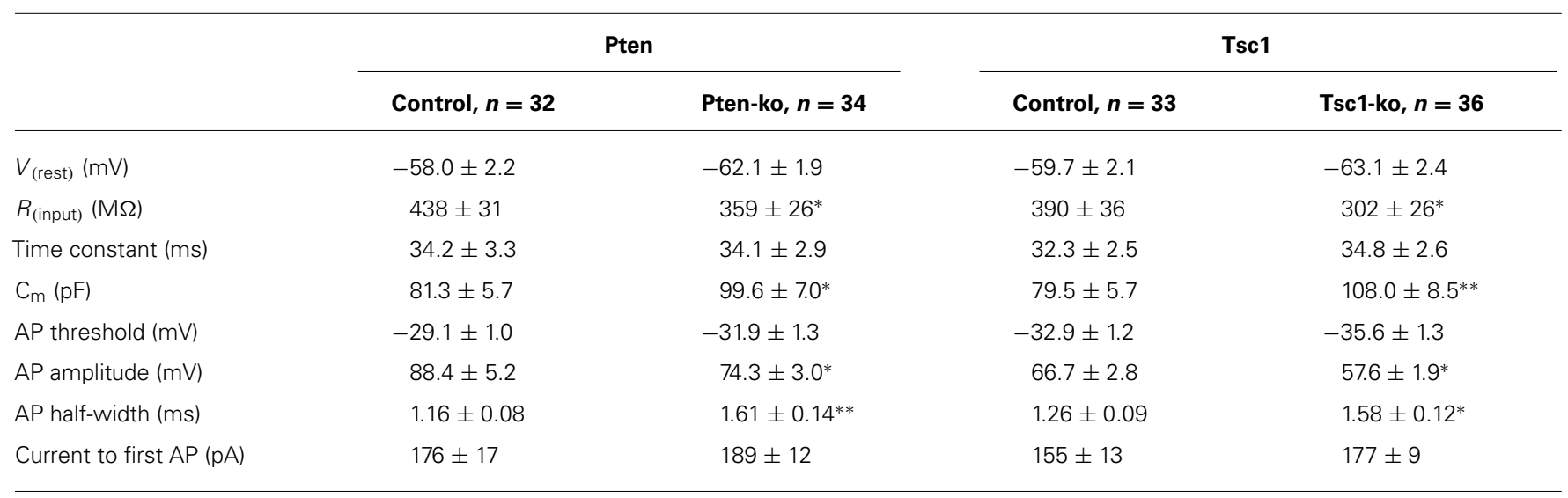

Measurements are shown as mean $\pm S E M ;{ }^{*} p \leq 0.05,{ }^{* *} p \leq 0.01$ compared to control with unpaired $t$-test or Mann-Whitney.

important contributor to the disease phenotypes (Wang et al., 2007; Luikart et al., 2011; Xiong et al., 2012). We compared the effects of Pten and Tsc1 loss on excitatory synaptic transmission using whole cell voltage clamp analysis of glutamatergic neurons. We began by evoking action potentials with a $2 \mathrm{~ms}$ depolarization to $0 \mathrm{mV}$ and recording the resulting EPSCs. Blockade of the EPSCs with $300 \mathrm{mM}$ kynurenic acid confirmed they were glutamatergic. In Pten-ko neurons, peak EPSC amplitudes were increased $70 \%$ relative to control neurons (Figures $\mathbf{3 A , B}$ ). The peak EPSC amplitudes of Tscl-ko neurons, however, were not different from control neurons (Figures 3A,B). The shape of the EPSCs in both Pten- and Tsc1-ko neurons, as measured by the rise and decay time constants, were unchanged relative to control (Figure 3C), indicating that the synchrony of vesicle release and the AMPA receptor subunit composition may be unchanged. 


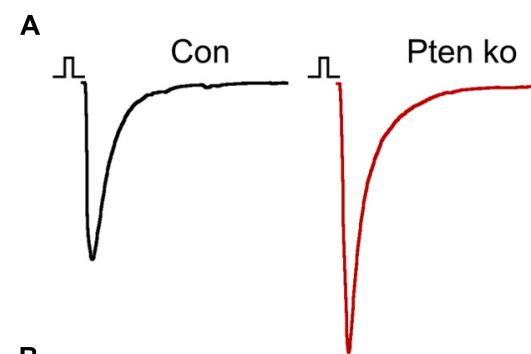

B
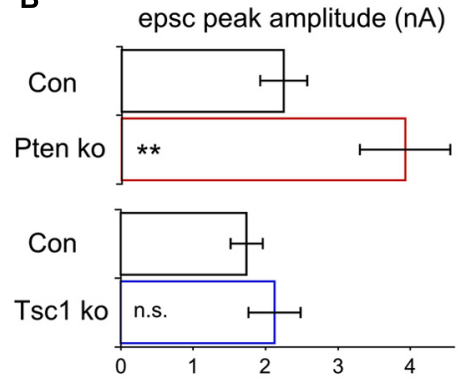

FIGURE 3 | Pten-ko neurons show increased AP-evoked EPSCs but Tsc1-ko neurons do not. (A) Representative responses of voltage-clamped Pten-ko (red trace) and Tsc1-ko (blue traces) neurons and their controls (black traces) to a $2 \mathrm{~ms}$ depolarization to $0 \mathrm{mV}$ from $-70 \mathrm{mV}$. (B) Bar graph showing AP-evoked EPSC peak amplitudes

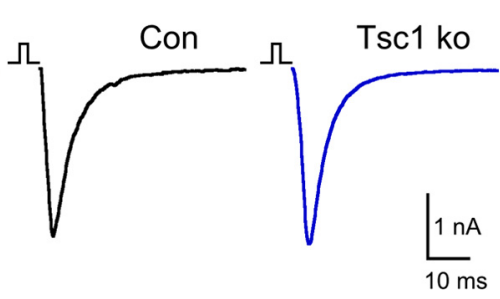

C

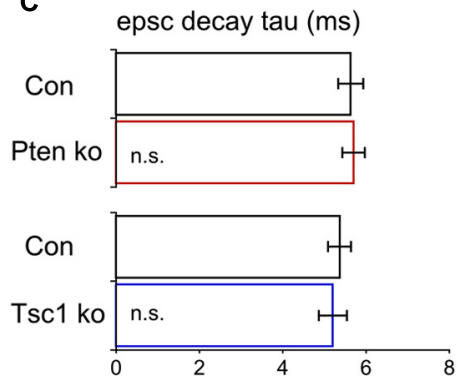

(mean \pm SEM) from Pten-ko (red bars) and Tsc1-ko (blue bars) and their respective controls (black bars). (C) Mean \pm SEM values for AP-evoked EPSC decay time constant from Pten-ko (red bars) and Tsc1-ko (blue bars) and their respective controls (black bars). ${ }^{* *} p \leq 0.01$, n.s. = not significant.

\section{LOSS OF Pten INCREASES MINIATURE EPSC TRANSMISSION BUT LOSS OF Tsc1 DOES NOT}

In theory, changes in several parameters could account for the increase in AP-evoked EPSC amplitude in Pten-ko neurons. These include: (1) miniature EPSC (mEPSC) amplitude, (2) total number of synapses or releasable synaptic vesicles, or (3) probability of vesicle release $\left(P_{\mathrm{vr}}\right)$. Even in the Tscl-ko neurons, increases in one of these parameters may be offset by a decrease in another. We therefore attempted to determine the contribution of each parameter to excitatory synaptic transmission in the Pten- and Tsc1-ko neurons.

Most prior studies of neurons lacking Pten have reported increases in miniature EPSC amplitude (Luikart et al., 2011; Weston et al., 2012; Xiong et al., 2012; but see, Sperow et al., 2012), while the results from Tsc1-ko neurons have been mixed (Tavazoie et al., 2005; Bateup et al., 2013). Under voltage clamp and in the presence of TTX, we recorded miniature events in glutamatergic hippocampal autaptic neurons (Figure 4). Pten-ko neurons had mEPSC peak amplitudes that were 25\% larger than control neurons, with similar decay time constants (Figures 4A,B). Tsc1-ko neurons' mEPSC amplitude and decay time constants, however, were not different from control (Figures 4A,B). We also determined the frequency of the miniature events. mEPSCs from Pten-ko neurons were not different from control, while the Tsc1-ko neurons had significantly reduced mEPSC frequency (Figures 4C,D). Based on our previous report on loss of Pten in granule neurons, the effects of Tsc1 loss and lack of effect of Pten loss on mini frequency likely arise from an inhibitory effect of loss on the rate constant for spontaneous vesicle release that is independent of changes in synapse number (Weston et al., 2012).

\section{LOSS OF Pten, BUT NOT Tsc1, INCREASES THE NUMBER OF RELEASABLE SYNAPTIC VESICLES BUT DOES NOT ALTER THE VESICULAR RELEASE PROBABILITY}

AP-evoked release in Pten-ko neurons is increased 70\%, while the mEPSC amplitude is increased only $25 \%$, suggesting that increases in other parameters may also contribute to the size of the EPSC. In addition, both Pten- and Tsc1-ko neurons have increased AP width (Figure 2C), which can lead to increased presynaptic calcium entry and vesicular release probability. Application of a hyperosmotic solution (500 mM sucrose) to neurons causes the release of all available synaptic vesicles in a calcium-independent manner, and can be used, in combination with the charge contained in the mEPSC, to determine the number of releasable synaptic vesicles and the probability of their release in response to an action potential (Rosenmund and Stevens, 1996; Fernandez-Chacon et al., 2001; Weston et al., 2011).

In Pten-ko neurons the current response to $500 \mathrm{mM}$ sucrose application was significantly increased relative to control, while in Tsc1-ko neurons it was not (Figure 5A). Next, for each neuron in the dataset, we calculated the total number of releasable synaptic vesicles by dividing the charge integral of the sucroseinduced current by the charge integral of the average mEPSC. Pten-ko neurons showed a $40 \%$ increase in the number of synaptic vesicles per neuron relative to control, while the number of synaptic vesicles in Tsc1-ko neurons was not different from control (Figure 5B, top bars). Then, by dividing the charge integral of the AP-evoked EPSC by the charge integral of the average mEPSC, we calculated the average number of synaptic vesicles released per AP (Figure 5B, bottom bars). Pten-ko neurons showed a $40 \%$ increase in the number of synaptic vesicles released per AP relative to control, while the number 
A
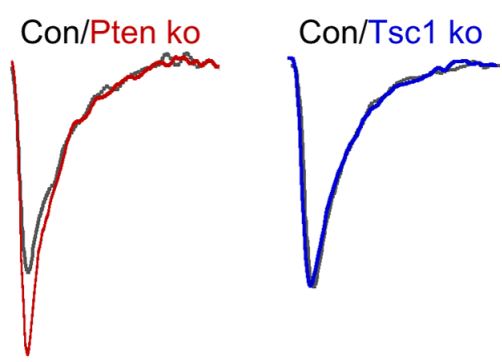

B

mini epsc peak amplitude $(p A)$

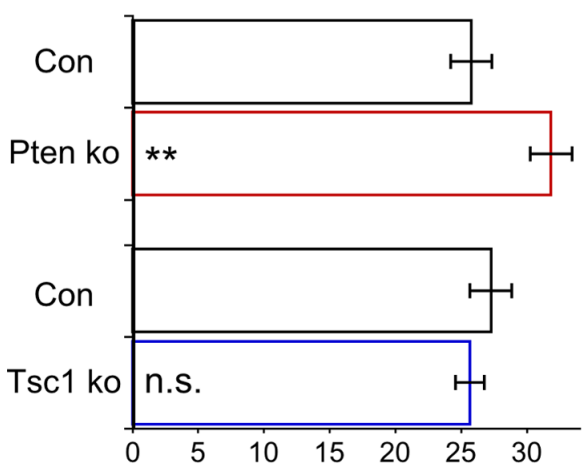

C
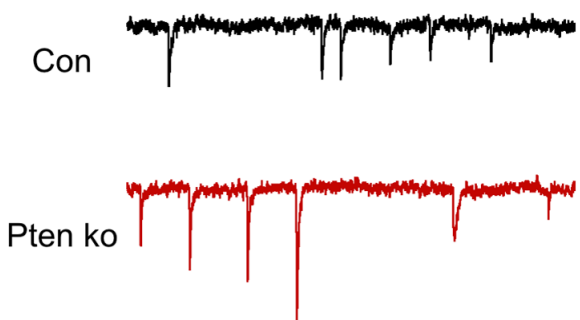

Tsc1 ko

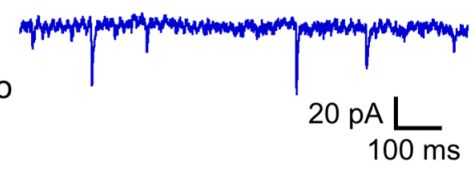

D

mini epsc frequency $(\mathrm{Hz})$

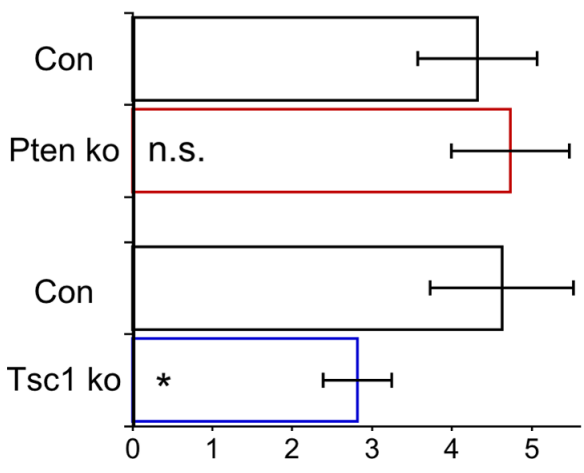

FIGURE 4 | Continued

\section{FIGURE 4 | Continued}

Pten-ko neurons show increased mEPSC amplitude but Tsc1-ko neurons do not. (A) Representative traces showing the average of mEPSCs collected from one Pten-ko neuron (red trace), one Tsc1-ko neuron (blue trace), and their respective controls (overlaid gray traces). (B) Bar graph showing mEPSC peak amplitude (mean \pm SEM) of Pten-ko (red bars) and Tsc1-ko (blue bars) neurons and their respective controls (black bars). (C) Representative traces showing control (black trace), Pten-ko (red trace), and Tsc1-ko (blue trace) miniature postsynaptic current activity from hippocampal glutamatergic neurons. (D) Bar graph showing mEPSC

frequency values (mean $\pm \mathrm{SEM}$ ) in $\mathrm{Hz}$ for Pten-ko (red bars) and Tsc1-ko (blue bars) neurons and their respective controls (black bars). ${ }^{*} p \leq 0.05$, ${ }^{* *} p \leq 0.01$, n.s. $=$ not significant.

of synaptic vesicles released per AP in Tscl-ko neurons was unchanged.

The vesicular release probability is the probability that an available vesicle can be exocytosed in response to $\mathrm{Ca}^{2+}$ influx during an action potential. This probability can be calculated as the ratio the number of vesicles released per AP to the total number of vesicles available for release. In both Pten- and Tsc1-ko neurons, the vesicular release probability was not different from control, supporting the conclusion that loss of these molecules does not greatly affect the efficiency of synaptic vesicle release (Figure 5C). Taken together, these results suggest that, in addition to mEPSC size, either an increased synapse number or number of vesicles per synapse may contribute to the EPSC increase seen with loss of Pten. However, these alterations are apparently absent in Tsc-ko neurons.

\section{LOSS OF Pten OR Tsc1 SHOWS SIMILAR EFFECTS ON DENDRITIC MORPHOLOGY BUT DIFFERENT EFFECTS ON SYNAPSE NUMBER}

Our electrophysiological analysis of Pten- and Tsc1-ko neurons showed that there was an increase in the number of releasable synaptic vesicles in Pten-ko neurons but not in Tsc1-ko neurons. The increase in the Pten-ko could be due to two non-exclusive possibilities: (1) An increase in the number of synaptic vesicles per synapse, or (2) an increase in the total number of synapses. To test this we immunostained our single neuron cultures for Vglut1, the synaptic vesicle protein that loads glutamate into vesicles and whose punctate staining pattern corresponds to excitatory synapses (Takamori et al., 2000; De Gois et al., 2006; Gabellec et al., 2007), and Map2 to visualize dendrites. We then imaged the neurons and reconstructed the dendritic trees using Neurolucida software and counted the number of VGlut1 positive punctae along the dendrites (Figure 6A). The total length of the dendrites was significantly increased in both mutants (Figures 6A,B), as was the number of branch points (Figure 6C). Control experiments showed that Map2 intensity in the soma was not elevated in Pten- or Tscl-ko neurons relative to control (97 $\pm 3 \%$ of control for Pten-ko, $95 \pm 5 \%$ of control for Tscl-ko) suggesting that the changes in dendrite morphology were not due to a general increase on Map2 levels due to mTOR activation. Next, we counted the number of Vglut1-positive punctae per neuron. Pten-ko neurons had a twofold increase in the number of Vglut1 punctae, while Tscl-ko neurons were unchanged compared to control (Figures 6D,E). Next, on a per neuron basis, we calculated the density of Vglut1 punctae. Pten-ko neurons had a 
A

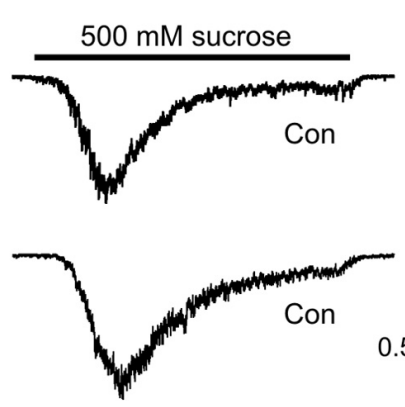

B

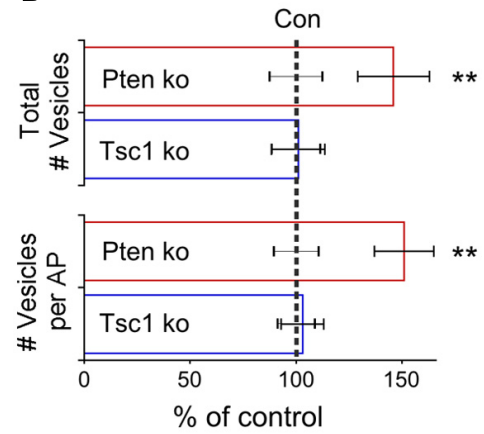

FIGURE 5 | Pten-ko neurons have increased total number of glutamatergic synaptic vesicles and released vesicles but Tsc1-ko neurons do not. (A) Representative traces showing the response to a $4 \mathrm{~s}$ application of $500 \mathrm{mM}$ sucrose from one Pten-ko neuron (red trace), one

Tsc1-ko neuron (blue trace), and their respective controls (black traces). (B) Bar graph showing the total number (mean \pm SEM) of glutamate-containing synaptic vesicles available for release in Pten-ko (top red bar) and Tsc1-ko (top

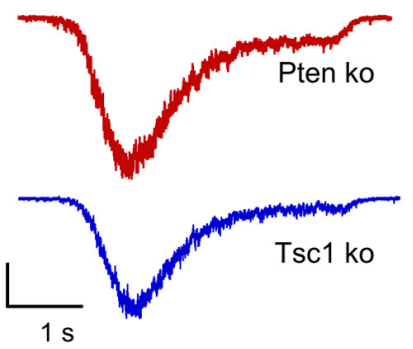

C

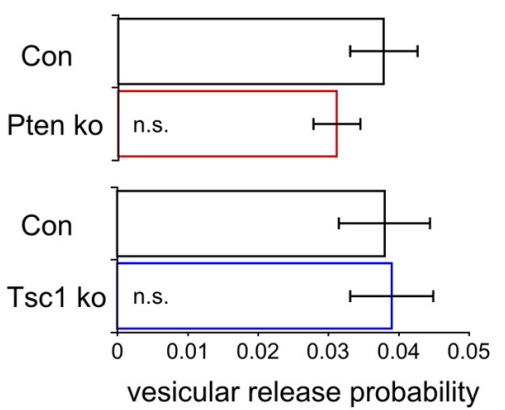

blue bar) neurons relative to their respective controls (black dashed line), as well as the average number (mean \pm SEM) of glutamate-containing synaptic vesicles released in response to an AP in Pten-ko (bottom red bar) and Tsc1-ko (bottom blue bar) neurons relative to their respective controls (black dashed line). (C) Bar graph showing the vesicular release probability (mean \pm SEM) of Pten-ko (red bar) and Tsc1-ko (blue bar) neurons relative to their respective controls (black bars). ${ }^{* *} p \leq 0.01$, n.s. $=$ not significant. non-significant increase in the density of Vglut1 punctae, while Tsc1-ko neurons had a significant decrease in the density of Vglut1 punctae (Figures 6D,F). Thus, Pten-ko neurons increase the number of their synaptic contacts in parallel with their increase in dendritic length and maintain synapse density. Tsc1-ko neurons, while also having a greater dendritic length, do not increase the number of synapses, resulting in a decrease in the overall density. Thus, loss of Pten and Tsc1 caused similar changes in the basic dendritic structure of single neurons, but the increased dendritic tree size is not sufficient to drive increases in excitatory synapse formation or neurotransmission, at least in Tsc1-ko neurons.

\section{LOSS OF Pten OR Tsc1 HAS OPPOSITE EFFECTS ON GABAergic NEUROTRANSMISSION}

Glutamatergic transmission was normal in Tsc1-ko neurons, and the current clamp analysis did not show any evidence for intrinsic hyperexcitability (Figure 2), however, network activity in Tsc1 deficient brains and cultures shows signs of hyperexcitability. We therefore tested whether the Pten and Tsc-ko neurons had any alterations in GABAergic transmission. We performed a whole cell voltage clamp analysis of single hippocampal GABAergic Ptenand Tsc1-ko neurons. A high-chloride intracellular solution was used so that GABA-gated chloride conductances elicited inward currents, and application of bicuculline verified the synaptic events were GABAergic. First, we recorded AP-evoked GABAergic synaptic responses in Pten- and Tscl-ko neurons (Figures 7A,B). In Pten-ko neurons, the mean peak amplitude of IPSCs was increased by $50 \%$, while in Tsc1-ko neurons the mean peak amplitude of IPSCs was decreased by $25 \%$ (Figures 7A,B). Next, we recorded miniature synaptic transmission. In Pten-ko neurons, the mean peak amplitude of mIPSCs was increased by $50 \%$, but the frequency was unchanged (Figures 7C-F). In Tsc1-ko neurons, the mean peak amplitude of mIPSCs was decreased by $30 \%$ (Figures 7C,D), while the frequency was unchanged (Figures 7E,F). These changes in miniature event size indicate that the changes in AP-evoked GABAergic transmission are due to reduced mini size, and most likely altered postsynaptic GABA receptor number.

Previous reports have suggested that the $B$ subunit of the $\mathrm{GABA}_{\mathrm{A}}$ receptor is a substrate of Akt (Wang et al., 2003; Xu et al., 2006). Akt phosphorylation of this subunit increases the amount of $\mathrm{GABA}_{\mathrm{A}}$ trafficked to the postsynapse and the amplitude of miniature IPSCs (mIPSC), and mediates an increase in GABAergic transmission in response to insulin (Wang et al., 2003; Xu et al., 2006; Luscher et al., 2011). Since loss of Pten and Tsc1 have been reported to have opposite effects on Akt activation, we immunostained our single neuron cultures for phospho-Akt 473 and quantified the effects of Pten and Tsc1 loss on fluorescence levels. Loss of Pten caused a $50 \%$ increase in the 
A

Con
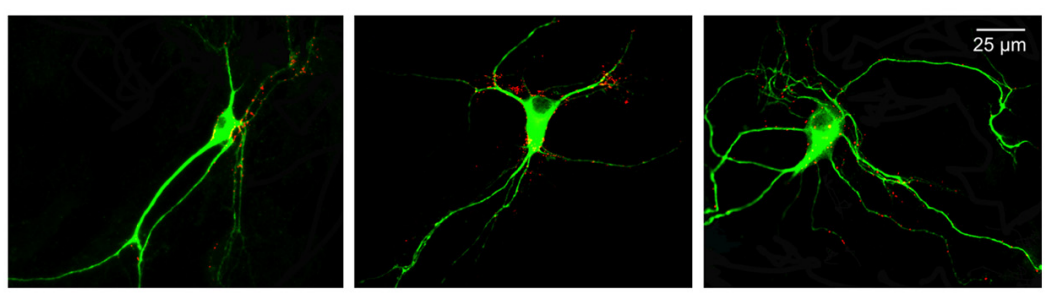

B

total dendritic length $(\mathrm{mm})$
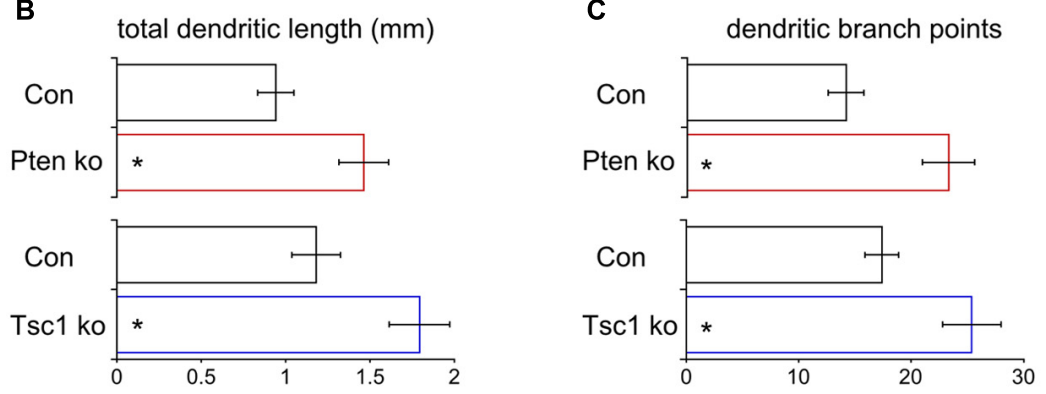

D

Con

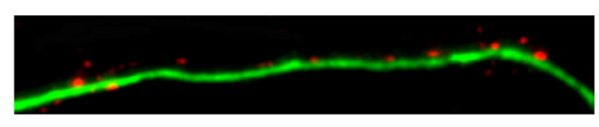

Pten ko

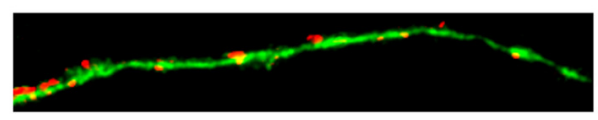

Tsc1 ko

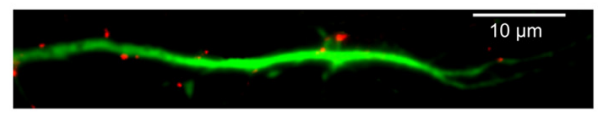

E
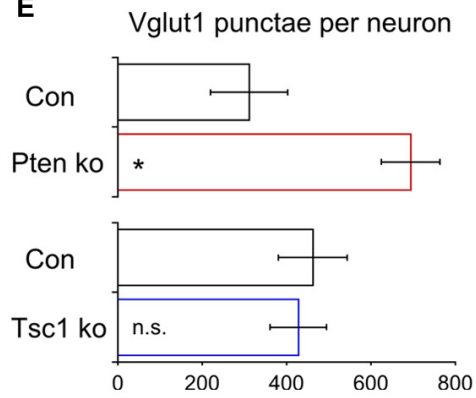

FIGURE 6 | Loss of Pten or Tsc1 has similar effects on dendrite growth but not on synapse number. (A) Representative images showing immunofluorescence from Map2 (green) and Vglut1 (red) of control, Pten-ko and Tsc1-ko neurons. (B) Bar graph showing total dendrite length (mean \pm SEM) of Pten-ko (red bars) and Tsc1-ko (blue bars) neurons and their respective controls (black bars). (C) Bar graph showing number of dendritic branch points (mean \pm SEM) of Pten-ko (red bars) and Tsc1-ko (blue bars) neurons and their respective controls (black

intensity of pAkt 473 fluorescence, while loss of Tsc1 caused a 40\% decrease in the intensity of pAkt473 fluorescence (Figures 7G,H), suggesting that the differential effect of Pten and Tscl loss on Akt activity may regulate inhibitory transmission in opposite directions.
$\mathbf{F}$
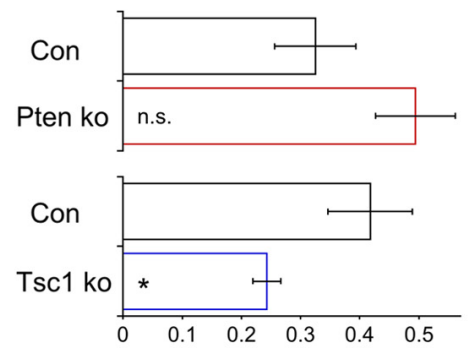

bars). (D) Representative images showing immunofluorescence of Map2 (green) and Vglut1 (red) on the dendrites of control, Pten-ko and Tsc1-ko neurons. (E) Bar graph showing total number of Vglut1 punctae (mean \pm SEM) per Pten-ko (red bars) and Tsc1-ko (blue bars) neuron and their respective controls (black bars). (F) Bar graph showing number of Vglut1 punctae per micron (mean \pm SEM) from Pten-ko (red bars) and Tsc1-ko (blue bars) neurons and their respective controls (black bars). $* p \leq 0.05$, n.s. $=$ not significant. 
A

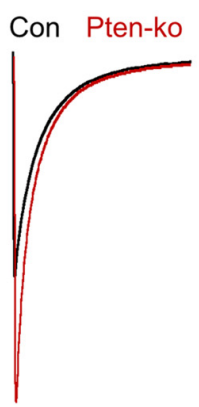

Con Tsc1-ko

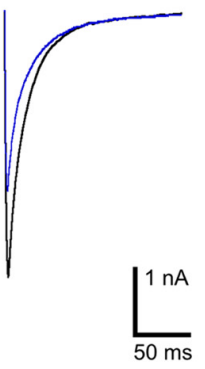

C

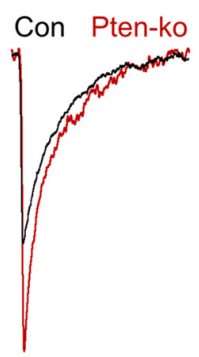

E

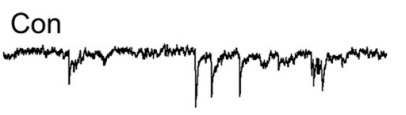

Pten-ko

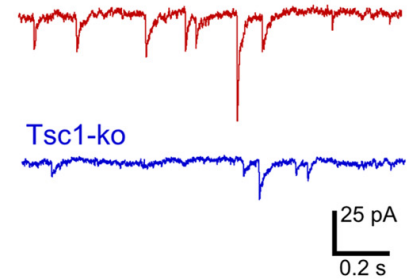

G
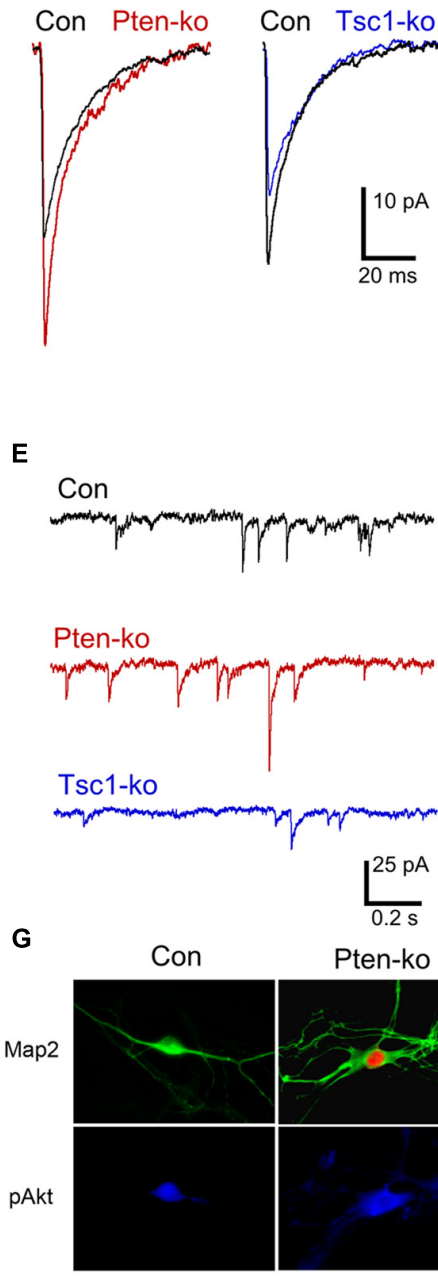

$0.2 \mathrm{~s}$
B

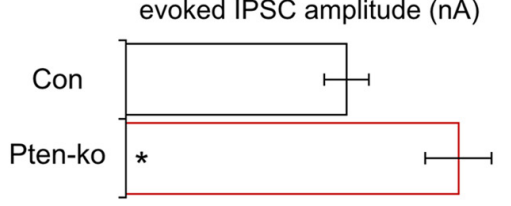

Con

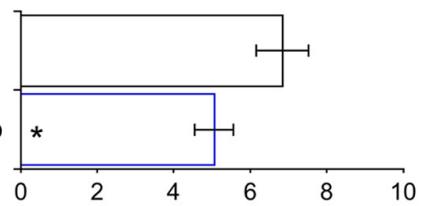

D

Con

Pten-ko

mIPSC peak amplitude $(\mathrm{pA})$

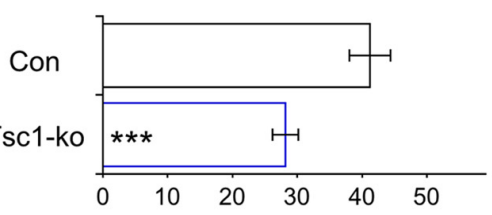

$\mathbf{F}$
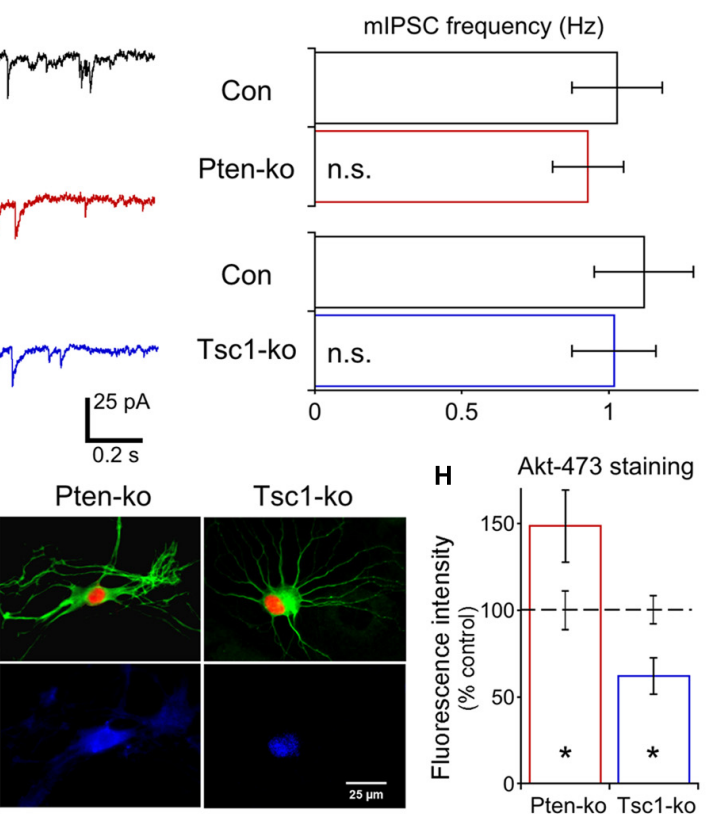

FIGURE 7 | Loss of Pten orTsc1 has opposite effects on GABAergic synaptic transmission in hippocampal GABAergic neurons. (A) Representative responses of voltage-clamped Pten-ko (red trace) and Tsc1-ko (blue traces) neurons and their controls (overlaid black traces) to a $2 \mathrm{~ms}$ depolarization to $0 \mathrm{mV}$ from $-70 \mathrm{mV}$. (B) Bar graph showing AP-evoked IPSC peak amplitudes (mean \pm SEM) of Pten-ko (red bars) and Tsc1-ko (blue bars) neurons and their respective controls (black bars). (C) Representative traces showing the average of mIPSCs collected from one Pten-ko neuron (red trace), one Tsc1-ko neuron (blue trace) and their respective controls (overlaid black traces). (D) Bar graph showing mIPSC peak amplitudes (mean \pm SEM) of Pten-ko (red bars) and Tsc1-ko (blue bars) neurons and their respective controls (black bars) (E) Representative traces showing control (black trace), Pten-ko (red trace), and Tsc1-ko (blue trace) miniature postsynaptic current activity from hippocampal GABAergic neurons. (F) Bar graph showing mIPSC frequencies (mean \pm SEM) of Pten-ko (red bars) and Tsc1-ko (blue bars) neurons and their respective controls (black bars). (G) Representative images showing immunofluorescence from Map2 (green) and p-Akt S473 (blue) from control (left column), Pten-ko (middle column) and Tsc1-ko (right column) neurons. Red fluorescence in Pten- and Tsc1-ko neurons is from the Cre-RFP fusion protein. (H) Intensity values (mean \pm SEM) for $p A k t$ measured from Pten-ko (red bar) and Tsc1-ko (blue bar) relative to their respective controls (black dashed line). ${ }^{*} p \leq 0.05, * * p \leq 0.01, * * * p \leq 0.001$, n.s. $=$ not significant. 
study in order to identify cellular phenotypes shared by neurons lacking Pten and Tsc1, reasoning that, since the disease phenotypes caused by loss of the two genes are similar, that the cellular phenotypes shared by the two might contribute to the organismal consequences of loss in a meaningful way. We expected, based on our previously published data on Pten, and on the hypothesis that alterations in the balance of excitation and inhibition in the brain may contribute to the development of both epilepsy and autism, that loss of either gene would lead to a direct increase in excitatory synaptic transmission.

In contrast to our original expectation, we found that only loss of Pten caused a direct increase in excitatory synaptic transmission, and that loss of Tsc1 had no measurable effect. Loss of Tsc1 did, however, cause a reduction in inhibitory synaptic transmission, an effect whose consequences may be functionally equivalent to an increase in excitatory transmission, especially in the context of the excitation-inhibition balance in a neuronal circuit. Other cellular effects of Pten and Tsc1 loss, such as the effects on neuronal growth, were similar, suggesting that these changes are likely important factors contributing to the disease phenotypes common to loss of Tscl or Pten.

\section{SIMILARITIES OF Pten AND Tsc1 LOSS}

Many of the molecular and cellular effects of Pten and Tsc1 loss were quantitatively similar. The majority of these changes have been described previously, including: (1) the increases in somatic and dendritic growth, (2) the alterations in membrane resistance and capacitance, and (3) the increases in levels of mTORC1 effectors pS6 and p4E-BP. Although our data showing changes in these parameters are not novel for loss of either gene, our study is the first to compare them side-by-side in the same experimental conditions. This is important for two reasons. First, the repeatability of the phenotypes gives further credence to the notion that they are direct consequences of Pten/Tsc1 loss and mTORC1 complex activation, and second, the side-by-side comparison shows that the magnitude of the changes caused by loss of either gene are comparable.

We also found that rapamycin treatment for $72 \mathrm{~h}$ caused a severe reduction in pS6 levels and normalized the soma area in both Pten- and Tscl-ko neurons, although it was ineffective at reducing $\mathrm{p} 4 \mathrm{E}-\mathrm{BP}$. The ability of rapamycin to inhibit the excessive S6 phosphorylation and cell growth in neurons caused by Pten and Tsc1 loss is well established, and there is also evidence that it can prevent increases in synaptic transmission caused by Pten loss (Weston et al., 2012; Xiong et al., 2012). The lack of an effect of long-term ( $>6 \mathrm{~h}$ ) rapamycin on phosphorylation of $4 \mathrm{E}-\mathrm{BP}$ and translation was previously shown to be cell-type dependent (Choo et al., 2008). Our data here showing that rapamycin does not decrease $\mathrm{p} 4 \mathrm{E}-\mathrm{BP}$ levels in hippocampal neurons caused by Pten or Tsc1 loss is therefore important to consider when interpreting the effects of rapamycin on neurons, neural circuits and the whole brain.

Although previously reported for loss of Pten (Weston et al., 2012), we show here that loss of Tscl also leads to a decrease in the amplitude and increase in the half-width of action potentials in hippocampal neurons. Interestingly, a recent study of neurons lacking FMRP also reported an increase in AP duration
(Deng et al., 2013). Loss of FMRP causes Fragile X syndrome, a disorder in which autism and epilepsy are frequently present, suggesting that altered presynaptic calcium influx due to changes in action potential shape could contribute to these phenotypes. We did not, however, detect any changes in the probability of synaptic vesicle release in Pten- and Tsc1-ko neurons, which would be expected in response to changes in presynaptic calcium influx. In addition, two previous studies in Tsc1-lacking neurons reported either no change (Bateup et al., 2013), or an increase in the AP amplitude and apparent decrease in the half-width of neurons lacking Tsc1 (Normand et al., 2013). Thus, the physiological impact of the changes in AP shape on the alterations in synaptic transmission that we observed remains unclear at this time.

Despite the fact that we found reduced input resistance and no change in the AP voltage threshold, we did not detect an increase in the amount of injected current required to elicit an AP (Figure 2; Table 1). There are at least two possibilities for this. First, the magnitudes of the changes we found in $R_{\mathrm{m}}$ were not large (20-25\% decrease), and there was a small but non-significant shift in the AP threshold toward more hyperpolarized values. Thus, any change in threshold current due to Pten or Tsc1 loss may be simply beyond our detection limit. Second, it is also possible that loss of Pten or Tscl causes changes in voltage gated conductances activated by the depolarization that compensate for the reduced $\mathrm{R}_{\mathrm{m}}$ to normalize the current threshold. Regardless of the effects of Pten or Tsc1 loss on current threshold, our data do not support the position that the hyperexcitability demonstrated by Pten- or Tsc1deficient networks is due to intrinsic increases in the excitability of glutamatergic neurons.

\section{DIFFERENCES OF Pten AND Tsc1 LOSS: SYNAPTIC TRANSMISSION}

Despite the similarities of Pten and Tsc1/2, especially in their influence on mTORC1 activity, we show here that their direct effects on synaptic transmission in hippocampal neurons are strikingly different. We show that loss of Pten causes a large increase in AP-evoked glutamatergic transmission due to the combined effect on the mEPSC size and the number of synapses, while loss of Tscl causes no change. For loss of Pten, this increase in excitatory transmission has support from several studies in cortical (Xiong et al., 2012), dentate granule (Luikart et al., 2011; Weston et al., 2012; Takeuchi et al., 2013), and now other hippocampal neuron types (CA1 and CA3, this study), although one previous study reported a decrease in CA1 fEPSPs (Fraser et al., 2008). While initial reports of Tsc1 loss in hippocampal pyramidal neurons suggested increased AP-evoked glutamatergic transmission (Tavazoie et al., 2005; Bateup et al., 2011), more recent studies, even from the same group, have not shown an increase in hippocampal (Bateup et al., 2013) or Purkinje neurons (Tsai et al., 2012). Our results support the more recent findings suggesting that loss of Tsc1 does not increase evoked glutamatergic transmission.

We found that neurons lacking Pten have increased mIPSC amplitude while those lacking Tsc1 have decreased mIPSC amplitude, and that the changes in mIPSC amplitude are mirrored by changes in AP-evoked IPSCs. The regulation of inhibitory synaptic transmission by mTOR has been studied far less than 
the regulation of excitatory transmission, but our results are supported by two previous studies. Striatal neurons lacking Pten showed increased mIPSC and AP-evoked IPSC amplitudes (Weston etal., 2012) and hippocampal pyramidal neurons lacking Tsc1 showed decreased mIPSC and evoked IPSC amplitudes (Bateup et al., 2013). However, shRNA-mediated knockdown of Pten has been reported not to produce a significant increase in IPSC amplitude in dentate granule neurons (Luikart et al., 2011).

In vivo, the parameters that regulate the synaptic input and output of a neuron are determined by intrinsic factors, such as its genetic program, and extrinsic factors, such as activity-dependent mechanisms. In the autapic neuron preparation that we use here, the synaptic properties are largely determined by intrinsic factors, as autaptic neurons do not exhibit classic mechanisms of plasticity such as LTP or homeostatic scaling (Chang et al., 2014). Although many of the changes in synaptic transmission due to Pten and Tsc1 loss in this work are similar to those seen in acute slice, some effects seen only in slice may be due to network-dependent effects, while others may be masked in slice due to network compensation. Understanding whether certain consequences of Pten or Tsc1 loss are cell autonomous or network dependent is important, however, and the comparison of the autaptic culture to acute slice and even in vivo data, if available, can help answer these questions.

\section{DIFFERENCES OF Pten AND Tsc1 LOSS: MOLECULAR EFFECTS}

In this report we show, as others previously, that the phosphorylation of Akt at S473 is increased by Pten loss and decreased by Tsc1 loss in the brain (Groszer et al., 2001; Kwon et al., 2001; Meikle et al., 2008; Ljungberg et al., 2009; Carson et al., 2012). Altered levels of phospho-Akt S473 would be expected to increase or decrease Akt activity toward its substrates (Sarbassov et al., 2005b). Previous reports have shown that knockdown of Akt with siRNA decreased glutamatergic synapse formation (Majumdar et al., 2011), and activating PI3K (analogous to loss of Pten in its effect on Akt phosphorylation) has been shown to increase the exocytosis of AMPA receptors and increase mEPSC amplitude (Man et al., 2003). While pharmacological inhibition of PI3K (analogous to loss of Tsc1 in effect on Akt phosphorylation) does not affect AMPA receptor insertion at baseline conditions (Man et al., 2003), it does block the insertion of AMPA receptors in response to synaptic stimulation and homeostatic compensation (Qin et al., 2005; Hou et al., 2008; Shehata et al., 2012). Thus, it is possible that activation of Akt in Pten-ko neurons leads to enhanced trafficking of AMPA receptor subunits to the synaptic membrane, which could be a critical step in increased synapse formation in these cells.

While the list of potential Akt substrates is long and the cellular effects of altered Akt activity are likely to be complex, the $ß$ subunit of the $\mathrm{GABA}_{\mathrm{A}}$ receptor stands out as particularly relevant to the effects on GABAergic transmission we report here. Since Akt phosphorylation of this subunit increases the amount of $\mathrm{GABA}_{\mathrm{A}}$ receptor trafficked to the synapse and the amplitude of mIPSCs (Wang et al., 2003; Xu et al., 2006; Luscher et al., 2011), it is possible that the changes in inhibitory synaptic transmission in response to Pten and Tsc1 loss are results of the effects on Akt activity.
While the differences in Akt activity may contribute to the effects on synaptic transmission, other factors are likely to be involved. The ability of Pten loss to increase excitatory neurotransmission could be independent of downstream mTOR signaling, and be due instead to Pten activity toward substrates other than $\mathrm{PIP}_{3}$. This hypothesis is attractive in its simplicity, however, the ability of rapamycin to prevent the increases in EPSCs strongly argues against this contention (Weston et al., 2012; Xiong et al., 2012). Nonetheless, Pten's protein phosphatase activity alone appears to be sufficient to decrease the number of dendritic spines in slice culture (Zhang et al., 2012), and Pten has been reported to bind to PSD-95 in response to NMDA receptor activation (Jurado et al., 2010), suggesting that alternative, postsynaptic targets may exist.

Taken together, our results suggest that excessive mTORC1 activity caused by loss of either Pten or Tsc1 is sufficient to drive increases in neuronal growth, but not increases in synapse formation and glutamatergic neurotransmission. However, our data also suggest that enhanced excitatory transmission may not be a necessary prerequisite to produce the disease phenotypes associated with hyperactivation of mTOR signaling, since loss of Tsc1-mediated suppression of synaptic inhibition may contribute to an overall excitatory-inhibitory imbalance.

\section{MATERIALS AND METHODS MICE AND CELL CULTURE}

Animal housing and use were in compliance with the National Institutes of Health (NIH) Guidelines for the Care and Use of Laboratory Animals and were approved by the institutional animal care committee at Baylor College of Medicine. Experiments utilized $\mathrm{Pten}^{\mathrm{tm} 1 \mathrm{Hwu}} / \mathrm{J}$ mice that possess loxP sites on either side of exon 5 of the PTEN gene, and Tsc1 ${ }^{\mathrm{tm} 1 \mathrm{Djk}} / \mathrm{J}$ mice that possess loxP sites preceding exon 17 and downstream of exon 18 (The Jackson Laboratory). The mice were backcrossed to C57BL/6 wild-type mice for at least six generations to ensure comparable backgrounds.

Microisland cultures of P0 hippocampal neurons were prepared according to published procedures (Reim etal., 2001; Weston et al., 2011, 2012). Microislands were made by coating collagen (0.7 $\left.\mathrm{mg} \mathrm{ml}^{-1}\right)$ and poly(D-lysine) $\left(0.1 \mathrm{mg} \mathrm{ml}^{-1}\right)$ on coverslips with a custom-built stamp to achieve uniform size (200 $\mu \mathrm{m}$ diameter). Astrocytes were grown on microislands for 1 week before plating of neurons. The hippocampus was removed from P0 mice of either sex. Neurons were then digested with papain (Worthington, Lakewood, NJ, USA) and plated on astrocytes derived from wild-type neonatal cortex tissue at a density of 2000-3000 neurons per $35 \mathrm{~mm}$ dish and grown in a chemically defined medium (Neurobasal-A medium supplemented with Glutamax and B-27; Invitrogen). Under these conditions, a single neuron on an astrocyte microisland forms recurrent synapses (autapses) (Bekkers and Stevens, 1991).

All experiments were performed on two to four independent cultures of neurons from either Pten ${ }^{\text {loxP}} /$ loxP or $\mathrm{Tsc}^{\text {loxP}} / \mathrm{loxP}$ mice infected with a lentivirus expressing a Cre recombinase-RFP fusion protein with a nuclear localization signal under control of the synapsin promoter 18-24 h after plating. For all experiments, two different types of control neurons were used, either Pten ${ }^{\text {loxP }} /$ loxP 
or Tsc1 ${ }^{\operatorname{lox} P / \operatorname{lox} P}$ infected with a control lentivirus expressing RFP at the same multiplicity of infection, or wild-type littermates of the experimental animals infected with the Cre recombinase-RFP fusion protein expressing lentivirus in the same amount. The values obtained from the two different control groups were not significantly different for any parameter tested and were therefore combined (see Table A1 in Appendix).

\section{ELECTROPHYSIOLOGY}

Standard extracellular solution contained the following (in $\mathrm{mM}$ ): $140 \mathrm{NaCl}, 2.4 \mathrm{KCl}, 10 \mathrm{HEPES}, 10$ glucose, $4 \mathrm{MgCl} 2$, and 2 $\mathrm{CaCl}$, pH 7.3 (305 mOsm). Internal solution contained the following: $136 \mathrm{mM} \mathrm{KCl}, 17.8 \mathrm{mM}$ HEPES, $1 \mathrm{mM}$ EGTA, $0.6 \mathrm{mM}$ $\mathrm{MgCl}$, $4 \mathrm{mM}$ ATP, $0.3 \mathrm{mM}$ GTP, $12 \mathrm{mM}$ creatine phosphate, and $50 \mathrm{U} / \mathrm{ml}$ phosphocreatine kinase. These concentrations set the chloride reversal potential high enough that GABA receptor mediated synaptic responses resulted in an inward current. All experiments were performed at room temperature $\left(23-24^{\circ} \mathrm{C}\right)$. Whole-cell recordings were performed on neurons from control and experimental groups in parallel on the same day in vitro (day 9-14 in vitro). Only neurons with visually identified expression of fluorescent reporters were targeted for patching.

For voltage clamp experiments, neurons were held at $-70 \mathrm{mV}$ unless noted. Action potential-evoked EPSCs or IPSCs were triggered by a $2 \mathrm{~ms}$ somatic depolarization to $0 \mathrm{mV}$. The shape of the evoked response and antagonists [either $3 \mathrm{mM}$ kynurenic acid (Tocris Bioscience) or $20 \mu \mathrm{M}$ bicuculline (Tocris Bioscience)] were applied to verify glutamatergic or GABAergic identities. Neurons were stimulated at $0.2 \mathrm{~Hz}$ (for EPSCs) or $0.1 \mathrm{~Hz}$ (for IPSCs) in standard external solution to measure basal evoked synaptic responses. The number of releasable synaptic vesicles of each neuron was determined by measuring the charge transfer of the transient synaptic current induced by a $4 \mathrm{~s}$ application of hypertonic sucrose solution directly onto the neuron and then dividing the sucrose charge by the charge of the average miniature event from the same neuron (Rosenmund and Stevens, 1996). To obtain $P_{\mathrm{vr}}$, the basal evoked synaptic responses and the response to the hypertonic sucrose solution were recorded successively from the same neuron. The evoked response was integrated to calculate the charge transfer. $P_{\mathrm{vr}}$ was calculated as the ratio of evoked response charge to RRP charge (Reim et al., 2001; Weston et al., 2011). For current clamp experiments, bias current was injected to achieve a resting membrane potential of $-70 \mathrm{mV}$ and kynurenic acid or bicuculline were applied to block synaptic responses. Input resistance and membrane time constant were calculated from the steady state and charging transient, respectively, of voltage responses to $0.5 \mathrm{~s}, 25 \mathrm{pA}$ hyperpolarizing current steps. Membrane capacitance was calculated by dividing the time constant by the input resistance. Action potentials (APs) were evoked with 0.5 s 25 pA depolarizing current steps. AP threshold was defined as $V_{\mathrm{m}}$ at the inflection point of the rising phase of the AP. AP amplitude was defined as the difference in $V_{\mathrm{m}}$ between the peak and threshold.

Data were analyzed offline with AxoGraph X 1.0 (AxoGraph Scientific, Sydney, Australia) and KaleidaGraph (Synergy Software, Reading, PA, USA). Values for analysis were always pooled from at least two independent cultures. Normality was tested by the Kolmogorov-Smirnov test. Statistical significances were tested by using Student's $t$ test, or the non-parametric Mann-Whitney test. One-way ANOVA followed SNK post-test was used when analyzing three groups.

\section{MINIATURE EVENT ANALYSIS}

Miniature synaptic potentials were recorded for 60-80 s with either $3 \mathrm{mM}$ kynurenic acid or $20 \mu \mathrm{M}$ bicuculline applied for $2 \mathrm{~s}$ of every $10 \mathrm{~s}$ for background noise subtraction and to verify the identity (glutamatergic or GABAergic) of the neuron under study. For some neurons, $500 \mathrm{nM}$ tetrodotoxin (TTX) was applied, however, there was no difference in the amplitude or frequency of events with or without TTX (Weston et al., 2012). For each cell, data were filtered at $1 \mathrm{kHz}$ and analyzed using template-based miniature event detection algorithms implemented in analysis software AxoGraph X 1.0. Threshold for detection was set at three times the baseline SD from a template of $0.5 \mathrm{~ms}$ rise time and $3 \mathrm{~ms}$ decay for glutamatergic events and 0.5 and $20 \mathrm{~ms}$ for GABAergic events.

\section{VIRUS PRODUCTION}

The preparation of lentiviral particles expressing Cre-RFP fusion protein or the RFP under control of the synapsin promoter was done as previously described (Lois et al., 2002). Briefly, HEK293T cells were co-transfected with $8 \mu \mathrm{g}$ shuttle vector F(SYN) UGWRBN bearing CDNA for cre-RFP, and the mixed helper plasmids pCMVdR8.9 and pVSV-G ( $5 \mu \mathrm{g}$ each) with Fugene 6 transfection reagent (Roche Diagnostics, Indianapolis, IN, USA). After 48 h the cell culture supernatant was collected and cell debris was removed by filtration with a $0.45 \mu \mathrm{m}$ polyethersulfone membrane (Pall Life Sciences, Ann Arbor, MI, USA). Aliquots of the filtrate were flash frozen in liquid nitrogen and stored at $-80^{\circ} \mathrm{C}$. Estimation of the titer was done on mass cultures of wild-type hippocampal neurons. For infection of the neurons for experiments $100 \mu \mathrm{l}$ of the viral solution $\left(0.9-1.8 \times 10^{6}\right.$ i.u./ml $)$ was used $18-24 \mathrm{~h}$ after plating.

\section{IMMUNOCYTOCHEMISTRY AND ANALYSIS}

After electrophysiology experiments were completed, neurons were rinsed with PBS and fixed in 4\% paraformaldehyde for $30 \mathrm{~min}$, then washed three times with PBS again. Samples were incubated in blocking buffer (5\% normal horse serum, $0.1 \%$ Triton X-100 in PBS) at room temperature for $1 \mathrm{~h}$. Primary antibodies diluted in blocking buffer were applied overnight at $4^{\circ} \mathrm{C}$. The following antibody dilutions were used: rabbit anti-phospho-S6 Ser 240/244 (1: 1,000, Cell Signaling Technology, Danvers, MA, USA), mouse anti-MAP2 (1:2000, Millipore/Chemicon, Temecula, CA, USA), rabbit anti-MAP2 (1:2000, Millipore/ Chemicon) rabbit anti-phospho-Akt (Ser473; 1: 1,000, Cell Signaling Technology), rabbit anti-phospho-4E-BP (Thr37/46; 1: 1,000, Cell Signaling Technology) guinea pig anti-VGLUT1 (1: 5,000, Synaptic Systems, Goettingen, Germany). After three washes for $5 \mathrm{~min}$ in PBS, secondary goat antibodies coupled to/conjugated with Alexa 488, 555, or 647 (Invitrogen/Molecular Probes, Eugene, OR, USA) were diluted 1:1000 in blocking buffer and applied for $1 \mathrm{~h}$ at room temperature. All images were obtained using Zeiss AxioImager M1 system with $40 \times$ Zeiss objectives and AxioVision software. 
For neuron reconstruction and analysis, images were acquired using equal exposure times and processed using the US National Institutes of Health ImageJ (http://rsbweb.nih.gov/ij/). A threshold macro was applied to the VGLUT channel to identify synapses. For cell body and dendrite tracing, the contrast of the images was increased so that the edges of the soma and the tips of dendrites were clearly visible to ensure the accuracy of the tracing. Neuron cell bodies, dendrites, and synapses were drawn using Neurolucida software (MicroBrightField, Williston, VT, USA). Quantification of the traced images was conducted with Neuroexplorer software (MicroBrightField). Raw values were then exported to KaleidaGraph (Synergy Software) for further analysis.

To quantify immunofluorescence intensity of pS6, p4E-BP, and pAkt, images of Map2 and the other antigen were acquired with equal exposure times and settings for control and experimental groups, assuring that none of the pixels were saturated. The images were then opened in Image J and the background was subtracted. Polygon selections were drawn around the cell body excluding the nucleus using the Map2 image. The average pixel intensity of the selected area in the pS6, p4E-BP, or pAkt image was then measured for each neuron.

\section{ACKNOWLEDGMENTS}

We would like to thank Dr. Amy Shore for critical reading of the manuscript. This work was supported by a postdoctoral fellowship from the Epilepsy Foundation (Matthew C. Weston) and by NIHNINDS grant NS018309 (John W. Swann).

\section{REFERENCES}

Avruch, J., Hara, K., Lin, Y., Liu, M., Long, X., Ortiz-Vega, S., et al. (2006). Insulin and amino-acid regulation of mTOR signaling and kinase activity through the Rheb GTPase. Oncogene 25, 6361-6372. doi: 10.1038/sj.onc.1209882

Backman, S. A., Stambolic, V., Suzuki, A., Haight, J., Elia, A., Pretorius, J., et al. (2001). Deletion of Pten in mouse brain causes seizures, ataxia and defects in soma size resembling Lhermitte-Duclos disease. Nat. Genet. 29, 396-403. doi: $10.1038 / \mathrm{ng} 782$

Bateup, H. S., Johnson, C. A., Denefrio, C. L., Saulnier, J. L., Kornacker, K., and Sabatini, B. L. (2013). Excitatory/inhibitory synaptic imbalance leads to hippocampal hyperexcitability in mouse models of tuberous sclerosis. Neuron 78, 510-522. doi: 10.1016/j.neuron.2013.03.017

Bateup, H. S., Takasaki, K. T., Saulnier, J. L., Denefrio, C. L., and Sabatini, B. L. (2011). Loss of Tscl in vivo impairs hippocampal mGluR-LTD and increases excitatory synaptic function. J. Neurosci. 31, 8862-8869. doi: 10.1523/JNEUROSCI.161711.2011

Bekkers, J. M., and Stevens, C. F. (1991). Excitatory and inhibitory autaptic currents in isolated hippocampal neurons maintained in cell culture. Proc. Natl. Acad. Sci. U.S.A. 88, 7834-7838. doi: 10.1073/pnas.88.17.7834

Carson, R. P., Van Nielen, D. L., Winzenburger, P. A., and Ess, K. C. (2012). Neuronal and glia abnormalities in Tscl-deficient forebrain and partial rescue by rapamycin. Neurobiol. Dis. 45, 369-380. doi: 10.1016/j.nbd.2011.08.024

Chang, C. L., Trimbuch, T., Chao, H. T., Jordan, J. C., Herman, M. A., and Rosenmund, C. (2014). Investigation of synapse formation and function in a glutamatergic-GABAergic two-neuron microcircuit. J. Neurosci. 34, 855-868. doi: 10.1523/JNEUROSCI.0229-13.2014

Choi, Y. J., Di Nardo, A., Kramvis, I., Meikle, L., Kwiatkowski, D. J., Sahin, M., et al. (2008). Tuberous sclerosis complex proteins control axon formation. Genes Dev. 22, 2485-2495. doi: 10.1101/gad.1685008

Choo, A. Y., Yoon, S. O., Kim, S. G., Roux, P. P., and Blenis, J. (2008). Rapamycin differentially inhibits S6Ks and 4E-BP1 to mediate cell-type-specific repression of mRNA translation. Proc. Natl. Acad. Sci. U.S.A. 105, 17414-17419. doi: 10.1073/pnas.0809136105

De Gois, S., Jeanclos, E., Morris, M., Grewal, S., Varoqui, H., and Erickson, J. D. (2006). Identification of endophilins 1 and 3 as selective binding partners for VGLUT1 and their co-localization in neocortical glutamatergic synapses: implications for vesicular glutamate transporter trafficking and excitatory vesicle formation. Cell Mol. Neurobiol. 26, 679-693. doi: 10.1007/s10571-006-9054-8

Deng, P. Y., Rotman, Z., Blundon, J. A., Cho, Y., Cui, J., Cavalli, V., et al. (2013). FMRP regulates neurotransmitter release and synaptic information transmission by modulating action potential duration via BK channels. Neuron 77, 696-711. doi: 10.1016/j.neuron.2012.12.018

Ehninger, D., De Vries, P. J., and Silva, A. J. (2009). From mTOR to cognition: molecular and cellular mechanisms of cognitive impairments in tuberous sclerosis. J. Intellect. Disabil. Res. 53, 838-851. doi: 10.1111/j.1365-2788.2009.01208.x

Feliciano, D. M., Quon, J. L., Su, T., Taylor, M. M., and Bordey, A. (2012). Postnatal neurogenesis generates heterotopias, olfactory micronodules and cortical infiltration following single-cell Tscl deletion. Hum. Mol. Genet. 21, 799-810. doi: $10.1093 / \mathrm{hmg} / \mathrm{ddr} 511$

Feliciano, D. M., Su, T., Lopez, J., Platel, J. C., and Bordey, A. (2011). Single-cell Tsc1 knockout during corticogenesis generates tuber-like lesions and reduces seizure threshold in mice. J. Clin. Invest. 121, 1596-1607. doi: 10.1172/JCI44909

Fernandez-Chacon, R., Konigstorfer, A., Gerber, S. H., Garcia, J., Matos, M. F., Stevens, C. F., et al. (2001). Synaptotagmin I functions as a calcium regulator of release probability. Nature 410, 41-49. doi: 10.1038/35065004

Fraser, M. M., Bayazitov, I. T., Zakharenko, S. S., and Baker, S. J. (2008). Phosphatase and tensin homolog, deleted on chromosome 10 deficiency in brain causes defects in synaptic structure, transmission and plasticity, and myelination abnormalities. Neuroscience 151, 476-488. doi: 10.1016/j.neuroscience.2007.10.048

Gabellec, M. M., Panzanelli, P., Sassoe-Pognetto, M., and Lledo, P. M. (2007). Synapse-specific localization of vesicular glutamate transporters in the rat olfactory bulb. Eur. J. Neurosci. 25, 1373-1383. doi: 10.1111/j.1460-9568.2007. 05400.x

Groszer, M., Erickson, R., Scripture-Adams, D. D., Lesche, R., Trumpp, A., Zack, J. A., et al. (2001). Negative regulation of neural stem/progenitor cell proliferation by the Pten tumor suppressor gene in vivo. Science 294, 2186-2189. doi: 10.1126/science. 1065518

Guertin, D. A., and Sabatini, D. M. (2009). The pharmacology of mTOR inhibition. Sci. Signal. 2, pe24. doi: 10.1126/scisignal.267pe24

Harris, T. E., and Lawrence, J. C. Jr. (2003). TOR signaling. Sci. STKE 2003, re15.

Hoeffer, C. A., and Klann, E. (2009). mTOR signaling: at the crossroads of plasticity, memory and disease. Trends Neurosci. 33, 67-75. doi: 10.1016/j.tins.2009.11.003

Hou, Q., Zhang, D., Jarzylo, L., Huganir, R. L., and Man, H. Y. (2008). Homeostatic regulation of AMPA receptor expression at single hippocampal synapses. Proc. Natl. Acad. Sci. U.S.A. 105, 775-780. doi: 10.1073/pnas.0706447105

Howell, J. J., and Manning, B. D. (2011). mTOR couples cellular nutrient sensing to organismal metabolic homeostasis. Trends Endocrinol. Metab. 22, 94-102. doi: 10.1016/j.tem.2010.12.003

Jaworski, J., Spangler, S., Seeburg, D. P., Hoogenraad, C. C., and Sheng, M. (2005). Control of dendritic arborization by the phosphoinositide-3'-kinaseAkt-mammalian target of rapamycin pathway. J. Neurosci. 25, 11300-11312. doi: 10.1523/JNEUROSCI.2270-05.2005

Jurado, S., Benoist, M., Lario, A., Knafo, S., Petrok, C. N., and Esteban, J. A. (2010). PTEN is recruited to the postsynaptic terminal for NMDA receptor-dependent long-term depression. EMBO J. 29, 2827-2840. doi: 10.1038/emboj.2010.160

Krueger, D. A., Wilfong, A. A., Holland-Bouley, K., Anderson, A. E., Agricola, K., Tudor, C., et al. (2013). Everolimus treatment of refractory epilepsy in tuberous sclerosis complex. Ann. Neurol. 74, 679-687. doi: 10.1002/ana.23960

Kwon, C. H., Luikart, B. W., Powell, C. M., Zhou, J., Matheny, S. A., Zhang, W., et al. (2006). Pten regulates neuronal arborization and social interaction in mice. Neuron 50, 377-388. doi: 10.1016/j.neuron.2006.03.023

Kwon, C. H., Zhu, X., Zhang, J., Knoop, L. L., Tharp, R., Smeyne, R. J., et al. (2001). Pten regulates neuronal soma size: a mouse model of Lhermitte-Duclos disease. Nat. Genet. 29, 404-411. doi: 10.1038/ng781

Laplante, M., and Sabatini, D. M. (2012). mTOR signaling in growth control and disease. Cell 149, 274-293. doi: 10.1016/j.cell.2012.03.017

Ljungberg, M. C., Sunnen, C. N., Lugo, J. N., Anderson, A. E., and D’Arcangelo, G. (2009). Rapamycin suppresses seizures and neuronal hypertrophy in a mouse model of cortical dysplasia. Dis. Model. Mech. 2, 389-398. doi: 10.1242/dmm.002386

Lois, C., Hong, E. J., Pease, S., Brown, E. J., and Baltimore, D. (2002). Germline transmission and tissue-specific expression of transgenes delivered by lentiviral vectors. Science 295, 868-872. doi: 10.1126/science.1067081 
Luikart, B. W., Schnell, E., Washburn, E. K., Bensen, A. L., Tovar, K. R., and Westbrook, G. L. (2011). Pten knockdown in vivo increases excitatory drive onto dentate granule cells. J. Neurosci. 31, 4345-4354. doi: 10.1523/JNEUROSCI.006111.2011

Luscher, B., Fuchs, T., and Kilpatrick, C. L. (2011). GABAA receptor trafficking-mediated plasticity of inhibitory synapses. Neuron 70, 385-409. doi: 10.1016/j.neuron.2011.03.024

Majumdar, D., Nebhan, C. A., Hu, L., Anderson, B., and Webb, D. J. (2011). An APPL1/Akt signaling complex regulates dendritic spine and synapse formation in hippocampal neurons. Mol. Cell. Neurosci. 46, 633-644. doi: 10.1016/j.mcn.2011.01.003

Man, H. Y., Wang, Q., Lu, W. Y., Ju, W., Ahmadian, G., Liu, L., et al. (2003). Activation of PI3-kinase is required for AMPA receptor insertion during LTP of mEPSCs in cultured hippocampal neurons. Neuron 38, 611-624. doi: 10.1016/S08966273(03)00228-9

Manning, B. D., and Cantley, L. C. (2003). United at last: the tuberous sclerosis complex gene products connect the phosphoinositide 3-kinase/Akt pathway to mammalian target of rapamycin (mTOR) signalling. Biochem. Soc. Trans. 31, 573-578. doi: 10.1042/BST0310573

Meikle, L., Pollizzi, K., Egnor, A., Kramvis, I., Lane, H., Sahin, M., et al. (2008). Response of a neuronal model of tuberous sclerosis to mammalian target of rapamycin (mTOR) inhibitors: effects on mTORC1 and Akt signaling lead to improved survival and function. J. Neurosci. 28, 5422-5432. doi 10.1523/JNEUROSCI.0955-08.2008

Meikle, L., Talos, D. M., Onda, H., Pollizzi, K., Rotenberg, A., Sahin, M., et al. (2007). A mouse model of tuberous sclerosis: neuronal loss of Tscl causes dysplastic and ectopic neurons, reduced myelination, seizure activity, and limited survival. J. Neurosci. 27, 5546-5558. doi: 10.1523/JNEUROSCI.554006.2007

Normand, E. A., Crandall, S. R., Thorn, C. A., Murphy, E. M., Voelcker, B., Browning, C., et al. (2013). Temporal and mosaic Tscl deletion in the developing thalamus disrupts thalamocortical circuitry, neural function, and behavior. Neuron 78, 895-909. doi: 10.1016/j.neuron.2013.03.030

Qin, Y., Zhu, Y., Baumgart, J. P., Stornetta, R. L., Seidenman, K., Mack, V., et al. (2005). State-dependent Ras signaling and AMPA receptor trafficking. Genes Dev. 19, 2000-2015. doi: 10.1101/gad.342205

Reim, K., Mansour, M., Varoqueaux, F., Mcmahon, H. T., Sudhof, T. C. Brose, N., et al. (2001). Complexins regulate a late step in $\mathrm{Ca} 2+$-dependent neurotransmitter release. Cell 104, 71-81. doi: 10.1016/S0092-8674(01) 00192-1

Rosenmund, C., and Stevens, C. F. (1996). Definition of the readily releasable pool of vesicles at hippocampal synapses. Neuron 16, 1197-1207. doi: 10.1016/S08966273(00)80146-4

Russo, E., Citraro, R., Constanti, A., and De Sarro, G. (2012). The mTOR signaling pathway in the brain: focus on epilepsy and epileptogenesis. Mol. Neurobiol. 46, 662-681. doi: 10.1007/s12035-012-8314-5

Sarbassov, D. D., Ali, S. M., and Sabatini, D. M. (2005a). Growing roles for the mTOR pathway. Curr. Opin. Cell Biol. 17, 596-603. doi: 10.1016/j.ceb.2005.09.009

Sarbassov, D. D., Guertin, D. A., Ali, S. M., and Sabatini, D. M. (2005b). Phosphorylation and regulation of Akt/PKB by the rictor-mTOR complex. Science 307, 1098-1101. doi: 10.1126/science.1106148

Shehata, M., Matsumura, H., Okubo-Suzuki, R., Ohkawa, N., and Inokuchi, K. (2012). Neuronal stimulation induces autophagy in hippocampal neurons that is involved in AMPA receptor degradation after chemical long-term depression. $J$. Neurosci. 32, 10413-10422. doi: 10.1523/JNEUROSCI.4533-11.2012

Sperow, M., Berry, R. B., Bayazitov, I. T., Zhu, G., Baker, S. J., and Zakharenko, S. S. (2012). Phosphatase and tensin homologue (PTEN) regulates synaptic plasticity independently of its effect on neuronal morphology and migration. J. Physiol. 590, 777-792.

Swiech, L., Perycz, M., Malik, A., and Jaworski, J. (2008). Role of mTOR in physiology and pathology of the nervous system. Biochim. Biophys. Acta 1784, 116-132. doi: 10.1016/j.bbapap.2007.08.015
Takamori, S., Rhee, J. S., Rosenmund, C., and Jahn, R. (2000). Identification of a vesicular glutamate transporter that defines a glutamatergic phenotype in neurons. Nature 407, 189-194. doi: 10.1038/35025070

Takeuchi, K., Gertner, M. J., Zhou, J., Parada, L. F., Bennett, M. V., and Zukin, R. S. (2013). Dysregulation of synaptic plasticity precedes appearance of morphological defects in a Pten conditional knockout mouse model of autism. Proc. Natl. Acad. Sci. U.S.A. 110, 4738-4743. doi: 10.1073/pnas.1222803110

Tavazoie, S. F., Alvarez, V. A., Ridenour, D. A., Kwiatkowski, D. J., and Sabatini, B. L. (2005). Regulation of neuronal morphology and function by the tumor suppressors Tsc1 and Tsc2. Nat. Neurosci. 8, 1727-1734. doi: 10.1038/ nn1566

Thoreen, C. C., Kang, S. A., Chang, J. W., Liu, Q., Zhang, J., Gao, Y., et al. (2009). An ATP-competitive mammalian target of rapamycin inhibitor reveals rapamycin-resistant functions of mTORC1. J. Biol. Chem. 284, 8023-8032. doi: 10.1074/jbc.M900301200

Tsai, P. T., Hull, C., Chu, Y., Greene-Colozzi, E., Sadowski, A. R., Leech, J. M., et al. (2012). Autistic-like behaviour and cerebellar dysfunction in Purkinje cell Tsc1 mutant mice. Nature 488, 647-651. doi: 10.1038/nature11310

Wang, Q., Liu, L., Pei, L., Ju, W., Ahmadian, G., Lu, J., et al. (2003). Control of synaptic strength, a novel function of Akt. Neuron 38, 915-928. doi: 10.1016/S0896-6273(03)00356-8

Wang, Y., Greenwood, J. S., Calcagnotto, M. E., Kirsch, H. E., Barbaro, N. M., and Baraban, S. C. (2007). Neocortical hyperexcitability in a human case of tuberous sclerosis complex and mice lacking neuronal expression of TSC1. Ann. Neurol. 61, 139-152. doi: 10.1002/ana.21058

Weston, M. C., Chen, H., and Swann, J. W. (2012). Multiple roles for mammalian target of rapamycin signaling in both glutamatergic and GABAergic synaptic transmission. J. Neurosci. 32, 11441-11452. doi: 10.1523/JNEUROSCI.128312.2012

Weston, M. C., Nehring, R. B., Wojcik, S. M., and Rosenmund, C. (2011). Interplay between VGLUT isoforms and endophilin Al regulates neurotransmitter release and short-term plasticity. Neuron 69, 1147-1159. doi: 10.1016/j.neuron.2011.02.002

Wullschleger, S., Loewith, R., and Hall, M. N. (2006). TOR signaling in growth and metabolism. Cell 124, 471-484. doi: 10.1016/j.cell.2006.01.016

Xiong, Q., Oviedo, H. V., Trotman, L. C., and Zador, A. M. (2012). PTEN regulation of local and long-range connections in mouse auditory cortex. J. Neurosci. 32, 1643-1652. doi: 10.1523/JNEUROSCI.4480-11.2012

Xu, E., Kumar, M., Zhang, Y., Ju, W., Obata, T., Zhang, N., et al. (2006). Intraislet insulin suppresses glucagon release via GABA-GABAA receptor system. Cell. Metab. 3, 47-58. doi: 10.1016/j.cmet.2005.11.015

Zhang, X. C., Piccini, A., Myers, M. P., Van Aelst, L., and Tonks, N. K. (2012). Functional analysis of the protein phosphatase activity of PTEN. Biochem. J. 444 457-464. doi: 10.1042/BJ20120098

Conflict of Interest Statement: The authors declare that the research was conducted in the absence of any commercial or financial relationships that could be construed as a potential conflict of interest.

Received: 08 November 2013; paper pending published: 21 November 2013; accepted: 09 January 2014; published online: 03 February 2014.

Citation: Weston MC, Chen H and Swann JW (2014) Loss of mTOR repressors Tsc1 or Pten has divergent effects on excitatory and inhibitory synaptic transmission in single hippocampal neuron cultures. Front. Mol. Neurosci. 7:1. doi: 10.3389/fnmol.2014. 00001

This article was submitted to the journal Frontiers in Molecular Neuroscience.

Copyright (C) 2014 Weston, Chen and Swann. This is an open-access article distributed under the terms of the Creative Commons Attribution License (CC BY). The use, distribution or reproduction in other forums is permitted, provided the original author(s) or licensor are credited and that the original publication in this journal is cited, in accordance with accepted academic practice. No use, distribution or reproduction is permitted which does not comply with these terms. 


\section{APPENDIX}

Table A1 | Comparison of the two different control groups for multiple measurements.

\begin{tabular}{|c|c|c|c|c|}
\hline & \multicolumn{2}{|c|}{ Pten } & \multicolumn{2}{|c|}{ Tsc1 } \\
\hline & Wild-type, cre-rfp virus & Pten flox/flox, rfp only virus & Wild-type, cre-rfp virus & Tsc1 ${ }^{\text {flox/flox }}$, rfp only virus \\
\hline pS6 intensity (a.u.) & $2959 \pm 530 n=9$ & $3247 \pm 417 n=9, p=0.7$ & $3453 \pm 530 n=7$ & $3780 \pm 571 n=10, p=0.6$ \\
\hline$C_{m}(p F)$ & $78.0 \pm 6.7 n=15$ & $84.0 \pm 8.1 n=17, p=0.6$ & $84.6 \pm 9.5 n=15$ & $74.2 \pm 5.9 n=18, p=0.4$ \\
\hline EPSC amp. (nA) & $1.81 \pm 0.27 n=16$ & $2.16 \pm 0.5 n=18, p=0.9$ & $1.69 \pm 0.27 n=17$ & $1.8 \pm 0.35 n=20, p=0.8$ \\
\hline mEPSC amp. (pA) & $24.1 \pm 2.9 n=15$ & $25.3 \pm 2.2 n=17, p=0.8$ & $26.4 \pm 2.8 n=13$ & $26.2 \pm 1.9 n=15, p=0.9$ \\
\hline RRP size (\# of vesicles) & $6852 \pm 1573 n=16$ & $6561 \pm 1684 n=18, p=0.7$ & $7550 \pm 1701 n=17$ & $8382 \pm 2144 n=20, p=0.8$ \\
\hline IPSC amp. (nA) & $6.29 \pm 0.6 n=15$ & $5.37 \pm 1.1 n=17, p=0.5$ & $6.66 \pm 1.6 n=17$ & $7.00 \pm 1.3 n=18, p=0.8$ \\
\hline mIPSC amp. (pA) & $35.7 \pm 4.6 n=15$ & $34.2 \pm 2.7 n=13, p=0.7$ & $43.3 \pm 4.8 n=14$ & $38.8 \pm 4.0 n=18, p=0.5$ \\
\hline
\end{tabular}

Measurements are shown as mean \pm SEM; $p$ value calculated with unpaired t-test or Mann-Whitney. 